\title{
Perspectives sur l'équipement de protection individuelle dans les établissements de soins actifs durant la pandémie de COVID-19
}

\author{
Josh Ng-Kamstra MDCM MSP, Henry T. Stelfox MD PhD, Kirsten Fiest PhD, John Conly MD \\ Jeanna Parsons Leigh PhD
}

Citation : CMAJ 2020 July 13;192:E805-59. doi : 10.1503/cmaj.200575-f; diffusion hâtive le 25 juin 2020

Voir la version anglaise de l'article ici : www.cmaj.ca/lookup/doi/10.1503/cmaj.200575

$\mathbf{E}$ $\mathrm{n}$ raison de la pandémie de maladie à coronavirus 2019 (COVID-19) qui continue de prendre de l'ampleur, les organisations de soins de santé ont dû sélectionner l'équipement de protection individuelle (EPI) pour les travailleurs de la santé qu'elles jugeaient approprié dans un contexte d'incertitude et de contraintes liées à la chaîne d'approvisionnement. Même si les experts de la prévention et du contrôle des infections ont formulé des conseils fondés sur des données probantes, beaucoup de travailleurs de la santé craignent d'être indûment exposés à un risque de transmission du coronavirus du syndrome respiratoire aigu sévère 2 (SRAS-CoV-2), le virus qui cause la COVID-19, en raison des politiques en place ou d'une pénurie d'équipement ${ }^{1}$. Des précautions contre la transmission par gouttelettes ou contact combinées à diverses activités, notamment l'hygiène des mains et le port de gants, de blouses, de lunettes de protection et de masques médicaux, sont généralement recommandées pour protéger les travailleurs contre les virus respiratoires². L'utilisation de masques N95 est généralement recommandée durant les interventions médicales générant des aérosols, comme l'intubation ${ }^{3}$.

Ces recommandations permettent-elles d'offrir aux travailleurs de la santé la protection contre la COVID-19 dont ils ont besoin? Nous analysons des perspectives sur l'EPI en milieu hospitalier afin d'orienter la prise de décisions et le dialogue durant la pandémie de COVID-19.

\section{Historique des recommandations actuelles sur l'utilisation d'EPI par les travailleurs de la santé dans le contexte de la COVID-19}

La transmissibilité et la virulence d'un virus respiratoire sont 2 facteurs à considérer au moment de sélectionner l'EPI à utiliser. Des virus respiratoires comme les rhinovirus ou les adénovirus causent généralement des infections de faible gravité, mais le SRAS-CoV-2 peut entraîner une maladie grave ou critique qui est associée à un risque de mortalité accru chez les personnes âgées

\section{POINTS CLÉS}

- Les lignes directrices sur l'utilisation de l'équipement de protection individuelle (EPI) pour les soins généraux prodigués aux patients atteints de la maladie à coronavirus 2019 (COVID19) dans les établissements de soins actifs recommandent le port de gants, d'une blouse d'isolement, d'une protection oculaire et d'un masque médical ou d'un masque N95.

- Compte tenu des caractéristiques connues de la transmission du coronavirus du syndrome respiratoire aigu sévère 2 (SRASCoV-2), le port d'un masque N95 est nécessaire seulement dans le cadre des interventions médicales générant des aérosols.

- Dans un contexte d'approvisionnement limité en masques N95, les organisations peuvent choisir parmi plusieurs stratégies qui visent à garantir la disponibilité de protections respiratoires en quantité suffisante, chaque stratégie ayant ses avantages et ses inconvénients.

- Les protocoles d'EPI plus complexes n'offrent pas nécessairement une meilleure protection, et certains peuvent même faire augmenter le risque d'autocontamination lors du retrait de l'équipement.

- Les hôpitaux peuvent adopter une approche de transfert intégré des connaissances pour la recherche et l'élaboration de politiques sur l'EPI afin d'instaurer la confiance, de s'assurer que les travailleurs de la santé croient en l'efficacité de leur EPI, et d'appliquer rapidement les nouvelles découvertes scientifiques dans la pratique.

et celles ayant des comorbidités ${ }^{4}$. Des décès ont été répertoriés parmi les travailleurs de la santé, bien qu'on observe des taux de létalité plus faibles parmi ce groupe que dans le reste de la population5. De plus, le taux de mortalité élevé associé à la COVID-19 au Canada s'explique par des éclosions dans des établissements de soins de longue durée, où les procédures de prise en charge des patients et la disponibilité de l'EPI varient grandement ${ }^{6}$. Compte tenu du risque de maladie grave et de propagation nosocomiale, d'importants efforts doivent être faits pour réduire le risque de transmission virale dans les établissements de soins de santé. 
Quand la COVID-19 a fait son apparition à Wuhan, en Chine, l'EPI utilisé pour traiter les patients était complexe et comportait de nombreux éléments. Dans le contexte de l'apparition d'un virus jusqu'alors inconnu et de l'information limitée qui existait sur sa transmissibilité, les travailleurs de la santé utilisaient 2 paires de gants, des tenues de protection complètes, des lunettes de protection, des bonnets de chirurgien et des masques N957. Malgré ces mesures, des milliers de travailleurs de la santé ont contracté le virus $^{8}$. Bon nombre de ces cas d'infection ont eu lieu au début de l'épidémie et la plupart d'entre eux étaient probablement d'origine communautaire ${ }^{5}$. Les lignes directrices des Centers for Disease Control and Prevention (CDC) des États-Unis ont ensuite suggéré que pour les soins généraux donnés aux patients ayant la COVID-19, l'EPI de base devait inclure 4 éléments : des gants, une blouse d'isolement, des lunettes de protection ou un écran facial

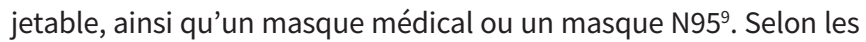
CDC, un masque N95 bien ajusté était préférable, mais en cas de pénurie, un masque médical suffisait pour les soins généraux. Au même moment, l'Organisation mondiale de la Santé et l'Agence de la santé publique du Canada recommandaient de réserver les masques N95 pour les interventions médicales générant des aérosols et d'utiliser des masques médicaux dans tous les autres contextes $^{10-12}$. L'Infectious Diseases Society of America a récemment publié des lignes directrices sur l'EPI fondées sur un outil appelé Grading of Recommendations Assessment, Development and Evaluation (GRADE), selon lesquelles le personnel peut utiliser un masque médical ou un masque N95 pour donner des soins aux patients atteints de la COVID-19 en l'absence d'interventions médicales générant des aérosols ${ }^{13}$.

\section{Dans la plupart des contextes, le SRAS-CoV-2 se transmet-il principalement par des gouttelettes ou par des aérosols?}

Les lignes directrices sur l'utilisation de l'EPI dépendent des modes de transmission. Les masques N95 sont recommandés comme protection contre les agents biologiques aérosolisés, tandis que les masques médicaux sont recommandés contre les gouttelettes $^{14}$. Les données probantes existantes sur les principaux modes de transmission du SRAS-CoV-2 soutiennent fortement la thèse de la transmission par gouttelettes et par contact ${ }^{13}$. On estime que la transmission par les aérosols ne joue pas un rôle important dans la plupart des situations. Des preuves indirectes appuieraient cette théorie : le $\mathrm{R}_{0}$ (nombre d'infections secondaires associées à chaque cas) des virus aérosolisés est généralement très élevé, s'élevant à plus de 10 dans le cas de la rougeole ${ }^{15}$. En comparaison, on estime que le $\mathrm{R}_{0}$ de la COVID-19 se situe entre 2,2 et 2,7, un taux comparable à celui de l'influenza et d'autres virus transmis par des gouttelettes ${ }^{16}$. La théorie de l'absence de transmission par les aérosols est également appuyée par le taux d'attaque, qui est relativement faible au sein des ménages : environ $10 \%$ des personnes qui côtoient des cas dans leur ménage deviennent infectées, un pourcentage beaucoup plus faible que celui auquel on s'attendrait dans le cas de la transmission par les aérosols ${ }^{17}$. Lorsque le virus est aérosolisé en laboratoire à l'aide d'un nébuliseur Collison à 3 jets, il peut demeurer sous forme aérosolisée pendant plusieurs heures. Cependant, ces conditions expérimentales ne reproduisent pas les interventions médicales générant des aérosols et ont peu de pertinence pour le milieu clinique ${ }^{18}$. Selon une étude réalisée en Chine, le matériel génétique du SRAS-CoV-2 était présent dans l'air dans 35\% des échantillons prélevés dans des services de soins intensifs, mais tous les tests sur des échantillons d'air étaient négatifs dans les établissements singapouriens et iraniens, et aucune étude n'a confirmé la présence de particules virales infectieuses dans l'air ${ }^{19-21}$.

\section{Quelles données probantes existe-t-il au sujet de l'efficacité des masques médicaux comparativement à celle des masques N95?}

Il existe une quantité importante de données sur les mesures nécessaires pour prévenir la transmission hospitalière du SRAS-CoV, le virus qui cause le SRAS. Selon des études de cas, le lavage des mains et le port de gants, de blouses, de protection oculaire et de masques médicaux ont été des facteurs importants de la prévention des infections chez les travailleurs de la santé à Hong Kong ${ }^{22,23}$. Cependant, les pratiques pour la prise en charge des patients atteints du SRAS en 2002-2003 ont varié d'un milieu à l'autre; dans le cas d'un établissement de Toronto, on a exigé le port de 2 paires de gants, de blouses, de bonnets, de lunettes de protection, de couvre-chaussures et d'un masque N95 bien ajusté24. Cependant, plusieurs documents publiés ont fait état de cas de transmission du SRAS-CoV à des travailleurs de la santé, même si ceux-ci utilisaient des masques N95 ${ }^{25,26}$.

Après l'éclosion du SRAS, un volume croissant de données probantes a montré que les masques médicaux offraient une protection comparable aux masques N95, en tant que composante de l'EPI utilisé durant les soins cliniques de routine, pour ce qui est de la prévention des infections respiratoires virales. Une revue systématique publiée en 2016 a mené à la conclusion que même si les masques N95 sont plus efficaces que les masques médicaux selon des mesures réalisées sur des substituts en contexte de laboratoire, les masques médicaux étaient tout aussi efficaces que les masques N95 pour la prévention des maladies respiratoires aiguës dans les milieux cliniques ${ }^{27}$. Selon un essai clinique randomisé de grande envergure publié en 2019, dans les milieux de soins ambulatoires, les masques médicaux parvenaient aussi efficacement que les masques N95 à prévenir les cas d'influenza confirmés en laboratoire ${ }^{28}$.

Une revue systématique des mesures visant à réduire la transmission du bétacoronavirus a comparé l'utilisation de masques N95 à la non-utilisation de masques et, séparément, l'utilisation de masques médicaux à la non-utilisation de masques ${ }^{29}$. Aucune des études n'était randomisée. Même si le risque global de transmission était plus faible dans les études qui incluaient des masques N95, aucune étude n'a directement comparé ceux-ci aux masques médicaux, ce qui signifie que les déclarations ${ }^{30}$ selon lesquelles les masques N95 sont supérieurs aux masques médicaux pour les soins généraux ne sont pas fondées. De plus, selon de nouvelles données, de nombreux travailleurs de la santé auraient été exposés sans le savoir à la COVID-19; la plupart d'entre eux utilisaient des masques médicaux plutôt que 
des masques N95, et la maladie aurait été transmise seulement dans les cas où le port du masque était intermittent ou omis ${ }^{31,32}$.

\section{Quelles interventions peuvent générer des aérosols?}

Les lignes directrices recommandent l'utilisation de masques N95 ou de respirateurs équivalents pour les soins administrés durant des interventions médicales générant des aérosols, même s'il y a des divergences quant aux interventions précises qui sont réellement considérées comme des interventions générant des aérosols ${ }^{13}$. D'après une revue systématique qui comprenait 10 études sur les interventions médicales générant des aérosols durant l'épidémie de SRAS, l'intubation trachéale, la ventilation non effractive, la trachéostomie et la ventilation manuelle avant l'intubation augmentaient systématiquement le risque pour les travailleurs de la santés3. D'autres interventions, comme les traitements par nébuliseur, la bronchoscopie et les compressions thoraciques, pouvaient également augmenter le risque. Les études incluses dans cette revue étaient considérées comme des études de faible qualité, mais il est peu probable que des données d'études randomisées ou prospectives soient publiées pour répondre à la question de savoir si des masques ou des protections respiratoires doivent être utilisés pour chaque intervention. Par conséquent, les listes des interventions médicales générales des aérosols qui sont contenues dans les lignes directrices ont tendance à être plus inclusives.

La question de savoir si les soins donnés à un patient des soins intensifs qui est intubé et branché à un appareil respiratoire à circuit fermé nécessitent le port d'un masque N95 mérite une attention particulière. Il faut tenir compte du risque qu'un tube de ventilateur soit accidentellement débranché; ce risque peut être plus élevé si le patient est souvent déplacé, par exemple pour recevoir une ventilation en position ventrale. Toutefois, même si un tube est débranché, aucune donnée probante n'indique que cet événement puisse mener à l'aérosolisation du virus. Les auteurs d'une étude menée à Taïwan durant l'épidémie du SRAS ont utilisé la réaction en chaîne de la polymérase pour détecter la présence du virus aérosolisé dans l'air autour d'un patient intubé; aucun virus n'a été détecté, même après l'extubation ${ }^{34}$. Malgré les restrictions de cette étude, qui portait sur un seul patient, celle-ci a confirmé dans une certaine mesure l'hypothèse que la transmission par les aérosols ne joue peut-être pas un rôle important durant les soins ordinaires donnés à un patient sous ventilation mécanique. Les lignes directrices de la Surviving Sepsis Campaign sur la COVID-19 recommandent l'utilisation de masques médicaux pour les patients atteints de la COVID-19 qui sont branchés à un appareil de ventilation à circuit fermé ${ }^{35}$.

\section{Approches pour la gestion d'un approvisionnement limité de masques N95}

Durant la pandémie de la nouvelle grippe $\mathrm{A}(\mathrm{H} 1 \mathrm{~N} 1)$, les fournisseurs de soins canadiens estimaient que l'EPI approprié était facilement accessible $^{36}$. Le contexte de la COVID-19 est très différent. Les masques N95 sont faits à l'aide d'une technologie plus sophisti- quée que les masques médicaux, ils coûtent plus cher et leurs stocks sont plus limités ${ }^{37}$. À la suite de la diminution drastique des exportations de la Chine et de l'augmentation de la demande mondiale de masques N95, ces produits sont devenus rares malgré la constitution de réserves après l'épidémie du SRAS 9038,39 .

Il y a un risque que les stocks de masques N95 à usage unique soient insuffisants pour durer jusqu'à la fin de la pandémie, malgré les mesures d'approvisionnement vigoureuses et novatrices prises par les gouvernements et les hôpitaux. Les politiques hospitalières peuvent donc adopter plusieurs approches :

1. Réserver l'utilisation des masques N95 aux interventions médicales générant des aérosols et recommander l'utilisation de masques médicaux pour les interactions comme les soins donnés aux patients intubés.

2. Rationner les stocks de masques $\mathrm{N} 95$ : certaines administrations ont limité le nombre de masques N95 à 1 masque par travailleur de la santé par jour, par semaine ou jusqu'à ce que le masque soit visiblement sale.

3. Réutiliser les masques N95 à l'aide de procédés de stérilisation novateurs.

4. Explorer l'utilisation de masques N95 réutilisables qui comprennent des cartouches ou des filtres remplaçables.

Chaque option a ses avantages et ses inconvénients. La première est une approche simple qui est appuyée par les lignes directrices de la Surviving Sepsis Campaign sur la COVID-19, mais qui pourrait ne pas être acceptée par l'ensemble des fournisseurs de soins de santé. La deuxième est également simple à appliquer, mais soulève des risques d'autocontamination à la suite de la réutilisation d'équipement conçu pour être jetable; les lignes directrices de l'Infectious Diseases Society of America tentent d'atténuer ce risque en suggérant que la protection respiratoire soit recouverte d'un écran facial ou d'un masque médical ${ }^{13}$. Pour ce qui est de la troisième option, l'Organisation mondiale de la Santé et les Centers for Disease Control and Prevention ont formulé des directives sur des options liées à l'utilisation prolongée ou à la réutilisation de masques, en tant que mesure temporaire en cas de pénurie d'EPI, si les autres stratégies ont été épuisées ${ }^{11,40}$. Un fabricant, $3 \mathrm{M}$, a reconnu l'utilisation de peroxyde d'hydrogène vaporisé comme méthode valide de recyclage des masques $\mathrm{N} 95^{41}$. Une étude récente en prépublication des National Institutes of Health indique qu'une bonne décontamination des masques N95 ne nuit pas à leur efficacité $^{42}$, et un important projet multicentrique est en cours afin de valider diverses méthodes de décontamination ${ }^{43}$. Enfin, le concept de respirateur réutilisable et lavable constitue une option attrayante, mais jusqu'à présent, elle n'a pas été utilisée dans des milieux cliniques et nécessitera vraisemblablement des procédures spécialisées de stérilisation et de réutilisation en milieu hospitalier ${ }^{44}$.

\section{Quels autres facteurs doivent orienter la sélection de l'EPI à utiliser dans les milieux de soins actifs?}

La configuration des unités peut influencer le type d'EPI sélectionné. Dans les hôpitaux où les patients atteints de la COVID-19 reçoivent des soins dans des unités distinctes (patients regroupés 
en cohortes), et où tous les soins peuvent être donnés sans retirer l'EPI, il est possible de choisir un EPI plus complexe, car la diminution du nombre de retraits de l'équipement réduit le risque accru d'autocontamination qui est associé au retrait d'EPI complexe ${ }^{45}$. Dans les unités où les patients atteints de la COVID19 sont soignés au même endroit que des patients non infectés, ou dans les unités où le personnel infirmier est tenu de retirer son équipement avant de vérifier les commandes ou de consigner les soins donnés, un EPI plus simple peut réduire le nombre d'erreurs lors du retrait de l'EPI et ainsi réduire le risque d'autocontamination.

Dans les unités où les patients sont regroupés en cohortes et où l'EPI est porté pendant de longues périodes, l'utilisation de masques N95 peut avoir des effets néfastes sur certains groupes de travailleurs de la santé; par exemple, il existe des données montrant une diminution des échanges gazeux et une augmentation de la charge métabolique chez les femmes enceintes qui utilisent des respirateurs ${ }^{46}$. De façon plus générale, des données indiquent que l'utilisation prolongée de respirateurs est associée à une augmentation de la résistance au flux d'air, ce qui mène à une diminution des volumes respiratoires, à l'hypercapnie, à une fatigue subjective et à des dermatites faciales ${ }^{47-50}$.

L'efficacité de l'EPI en conditions réelles peut varier, surtout durant les premières phases de son adoption. Les taux d'autocontamination peuvent être élevés jusqu'à ce que les travailleurs de la santé apprennent à utiliser en toute sécurité une nouvelle technique de port et de retrait de l'EPI ${ }^{51}$. Cet apprentissage n'est pas automatique, et il faut intégrer dans les processus de travail du temps pour l'enseignement, l'apprentissage, l'utilisation et le renforcement. La rapidité avec laquelle les nouvelles techniques d'utilisation de l'EPI peuvent être enseignées en toute sécurité demeure inconnue. Des protocoles d'EPI complexes ont été enseignés, répétés et mis en œuvre dans le contexte de la COVID-19 en Chine $^{7}$, ainsi que durant l'éclosion du virus Ebola en 2014-2015 ${ }^{45,51}$. Une plus grande quantité d'équipement n'est pas nécessairement meilleure : dans le cas de l'Ebola, par exemple, les erreurs critiques étaient plus fréquentes lors de l'utilisation de protocoles d'EPI complexes par rapport aux protocoles de base, et ce, malgré la formation ${ }^{45}$. De plus, le temps nécessaire pour revêtir et retirer l'équipement est plus long quand des protocoles plus complexes sont imposés ${ }^{45}$. L'augmentation du nombre d'erreurs lors de l'enfilage ou du retrait de l'équipement dans le cas d'un protocole complexe qui consistait à désinfecter les protections respiratoires à l'aide d'air propulsé a également été observée dans un contexte expérimental au Canada ${ }^{52}$. Le jumelage à une personne (un « compagnon ») qui supervise les procédures de retrait d'équipement et corrige les erreurs en temps réel peut protéger les travailleurs de la santé contre l'autocontamination, notamment dans le cas des protocoles d'EPI plus complexes ${ }^{14}$.

\section{Pourquoi faut-il tenir compte des perspectives des travailleurs de la santé dans les décisions organisationnelles sur l'EPI?}

L'une des questions fondamentales, mais rarement explorées, dans le choix des protocoles d'EPI à adopter est le degré auquel les travailleurs de la santé se sentent protégés par le protocole désigné par leur hôpital local ${ }^{53}$. Les personnes et les organisations peuvent interpréter la documentation scientifique différemment et peuvent arriver à différentes conclusions quant à l'EPI qu'il convient d'utiliser pour se protéger d'un nouveau virus dans un contexte donné. Un effondrement de la confiance et des communications peut mener à des conflits, à de l'anxiété et à des absences du travail. Par exemple, en mars 2020, certaines infirmières et certains groupes de soins infirmiers au Canada ont invoqué les lois sur la santé et la sécurité au travail pour refuser de travailler sans masques N95 parce qu'ils estimaient qu'il n'était pas sécuritaire de travailler sans eux ${ }^{54}$. De plus, le Syndicat canadien de la fonction publique a insisté pour dire que tous les employés des hôpitaux devraient avoir le choix de porter des masques $\mathrm{N} 95^{55}$, malgré l'existence de données probantes de qualité qui indiquent qu'ils ne sont pas nécessaires dans la plupart des milieux.

Manifestement, une communication synthétisée et simplifiée des données probantes qui sous-tendent les décisions organisationnelles sur l'utilisation de l'EPI, ainsi que des possibilités de consultation et de rétroaction pour les travailleurs de la santé sont des éléments essentiels, et ces activités devraient être guidées par les principes bien définis du transfert des connaissances ${ }^{56,57}$. L'article de 2005 d'Upshur et de ses collègues sur les facteurs éthiques à considérer dans le cadre de la planification en cas de pandémie met l'accent sur la transparence et la nécessité de permettre aux personnes concernées de remettre en question la justification des décisions ${ }^{58}$. Quand il faut prendre des décisions dans un contexte d'incertitude, il est d'importance primordiale de communiquer honnêtement ce qui est connu et ce qui est inconnu.

La Fédération canadienne des syndicats d'infirmières et d'infirmiers soutient qu'il faudrait faire appel à l'expertise du personnel infirmier pour réaliser des évaluations des risques en contexte clinique ${ }^{59}$. La mobilisation d'experts de plusieurs disciplines plus tôt dans le processus - dans le cadre de la planification en cas de pandémie et de la rédaction des lignes directrices en matière de santé et de sécurité au travail - optimise la collaboration des professionnels de la santé et permet une analyse plus approfondie de la documentation scientifique que l'analyse qu'il est possible de faire en contexte clinique. Cette concertation peut aussi servir à ajouter un aspect pragmatique aux politiques de contrôle des infections. D'ailleurs, ce rôle de planification est inscrit dans le Code de déontologie de l'Association des infirmières et infirmiers du Canada ${ }^{60}$.

Une telle mobilisation des "usagers des connaissances », de la conception jusqu'à la mise en œuvre des politiques, est un aspect fondamental du transfert intégré des connaissances. Les Instituts de recherche en santé du Canada soulignent la nature dynamique et itérative de l'application des connaissances intégrée $^{61}$; ces caractéristiques font en sorte que cette méthode est parfaitement adaptée au contexte en évolution rapide d'une pandémie. En plaçant les perspectives des travailleurs de la santé au cœur de l'élaboration des politiques, les organisations peuvent instaurer la confiance, améliorer les communications et veiller à ce que les nouvelles recommandations soient rapidement appliquées. 


\section{Conclusion}

Les lignes directrices sur l'utilisation de l'EPI se fondent sur notre compréhension actuelle du mode de transmission du SRAS-CoV-2 et fournissent un point de départ pour les stratégies de prévention et de contrôle des infections nosocomiales durant cette pandémie ${ }^{11}$. Pour protéger les travailleurs de la santé du SRAS-CoV-2 et des pathogènes associés à des pandémies futures, il est essentiel de répondre à plusieurs questions, à savoir comment les aérosols sont générés, comment il faut gérer un approvisionnement en EPI limité, comment les soins peuvent être organisés pour optimiser l'utilisation de l'EPI et comment les perspectives des travailleurs de la santé peuvent être prises en compte dans les décisions organisationnelles. En raison de la probabilité que ce virus continue de circuler pendant des mois, voire des années, il est plus urgent et plus important que jamais de mener des recherches supplémentaires. En ayant une meilleure compréhension de la science fondamentale du comportement des aérosols dans les milieux de la santé, en investissant dans des technologies perturbatrices qui améliorent l'EPI et en encourageant l'innovation dans les systèmes et les cheminements de soins pour les patients atteints de maladies infectieuses, il serait possible de mieux protéger à la fois les patients et les travailleurs de la santé. Enfin, en utilisant une approche de transfert intégré des connaissances pour l'élaboration des politiques qui fait participer les travailleurs de la santé à la planification et à l'évaluation des stratégies en matière d'EPI, il sera possible de veiller à ce que les personnes qui utilisent l'EPI aient confiance en cet équipement et l'utilisent efficacement.

\section{Références}

1. Thielking M. Frustrated and afraid about protective gear shortages, health workers are scouring for masks on their own. STAT news 2020 Mar. 18.

2. Guidance: infection prevention and control measures for healthcare workers in acute care and long-term care settings. Ottawa: Public Health Agency of Canada; 2010. Accessible ici : https://ipac-canada.org/photos/custom/OldSite/ pdf/101217newsEN_Final_Seasonal_Influenza-1.pdf (consulté le 18 mars 2020).

3. Prevention strategies for seasonal influenza in healthcare settings: guidelines and recommendations. Atlanta: Centers for Disease Control and Prevention; 2018. Accessible ici : www.cdc.gov/flu/professionals/ infectioncontrol/healthcaresettings.htm (consulté le 18 mars 2020).

4. Wu Z, McGoogan JM. Characteristics of and important lessons from the coronavirus disease 2019 (COVID-19) outbreak in China: summary of a report of 72314 cases from the Chinese Center for Disease Control and Prevention. JAMA 2020 Feb. 24 [Cyberpublication avant impression]. doi: 10.1001/ jama.2020.2648.

5. Report of the WHO-China Joint Mission on coronavirus disease 2019 (COVID19). Geneva: World Health Organization; 2020.

6. Fisman D, Bogoch I, Lapointe-Shaw L, et al. Failing our most vulnerable: COVID-19 and Long-Term Care Facilities in Ontario. medRxiv 2020 Apr. 17. doi: 10.1101/2020.04.14.20065557.

7. The First Affiliated Hospital - Zhejiang University School of Medicine. Handbook of COVID-19 prevention and treatment. Hangzhou (China) : The Jack Ma Foundation and Alibaba Foundation; 2020. Accessible ici : gmcc.alibabadoctor. com/prevention-manual/detail?content_id=0 (consulté le 18 mars 2020).

8. Wang J, Zhou M, Liu F. Reasons for healthcare workers infected with novel coronavirus disease 2019 (COVID-19) in China. J Hosp Infect 2020;105:100-1.

9. Interim infection prevention and control recommendations for patients with suspected or confirmed coronavirus disease 2019 (covid19) in healthcare settings. Atlanta: Centers for Disease Control and Prevention; 2020. Accessible ici : www.cdc.gov/[le] coronavirus/2019-ncov/infection-control/control -recommendations.html
10. Coronavirus disease (COVID-19) technical guidance: infection prevention and control / WASH. Geneva: World Health Organization; 2020. Accessible ici : www.who.int/emergencies/diseases/novel-coronavirus-2019/technical-guidance /infection-prevention-and-control (consulté le 18 mars 2020).

11. Rational use of personal protective equipment for coronavirus disease (COVID-19) and considerations during severe shortages: interim guidance. Geneva: World Health Organization; 2020.

12. Infection prevention and control for COVID-19: second interim guidance for acute healthcare settings. Ottawa: Public Health Agency of Canada; 2020. Accessible ici : www.canada.ca/en/public-health/services/diseases/2019-novel -coronavirus-infection/health-professionals/infection-prevention-control-covid -19-second-interim- guidance.html\#a8.7 (consulté le 20 mai 2020).

13. Lynch J, Davitkov P, Anderson D, et al. Infectious Diseases Society of America Guidelines on infection prevention for health care personnel caring for patients with suspected or known COVID-19. Arlington (Va) : Infectious Diseases Society of America; 2020. Accessible ici : www.idsociety.org/practice -guideline/covid-19-guideline-infection-prevention (consulté le 17 mai 2020).

14. Conly J, Eremin S, Pessoa-Silva C, et al., editors. Infection prevention and control of epidemic- and pandemic-prone acute respiratory infections in health care. Geneva: World Health Organization; 2014.

15. Guerra FM, Bolotin S, Lim G, et al. The basic reproduction number (R0) of measles: a systematic review. Lancet Infect Dis 2017;17:e420-8.

16. Sanche S, Lin YT, Xu C, et al. High contagiousness and rapid spread of severe acute respiratory syndrome coronavirus 2. Emerg Infect Dis 2020;26(7).

17. Burke RM, Midgley CM, Dratch A, et al. Active monitoring of persons exposed to patients with confirmed COVID-19 - United States, January-February 2020. MMWR Morb Mortal Wkly Rep 2020;69:245-6.

18. van Doremalen N, Bushmaker T, Morris DH, et al. Aerosol and surface stability of SARS-CoV-2 as compared with SARS-CoV-1. N Engl J Med 2020;382:1564-7.

19. Ong SWX, Tan YK, Chia PY, et al. Air, surface environmental, and personal protective equipment contamination by severe acute respiratory syndrome coronavirus 2 (SARS-CoV-2) from a symptomatic patient. JAMA 2020;323:1610-2.

20. Guo ZD, Wang ZY, Zhang SF, et al. Aerosol and surface distribution of severe acute respiratory syndrome coronavirus 2 in hospital wards, Wuhan, China, 2020. Emerg Infect Dis 2020;26(7).

21. Faridi S, Niazi S, Sadeghi K, et al. A field indoor air measurement of SARS-CoV-2 in the patient rooms of the largest hospital in Iran. Sci Total Environ 2020; 725:138401.

22. Lau JT, Fung KS, Wong TW, et al. SARS transmission among hospital workers in Hong Kong. Emerg Infect Dis 2004;10:280-6.

23. Seto WH, Tsang D, Yung RW, et al. Effectiveness of precautions against droplets and contact in prevention of nosocomial transmission of severe acute respiratory syndrome (SARS). Lancet 2003;361:1519-20.

24. Cheng VC, Chan JF, To KK, et al. Clinical management and infection control of SARS: lessons learned. Antiviral Res. 2013;100:407-19.

25. Cluster of severe acute respiratory syndrome cases among protected healthcare workers - Toronto, Canada, April 2003. MWR Morb Mortal Wkly Rep 2003;52:433-6.

26. Chen YC, Chen PJ, Chang SC et al. Infection control and SARS transmission among healthcare workers, Taiwan. Emerg Infect Dis 2004;10:895-8.

27. Smith JD, MacDougall CC, Johnstone J, et al. Effectiveness of N95 respirators versus surgical masks in protecting health care workers from acute respiratory infection: a systematic review and meta-analysis. CMAJ 2016;188:567-74.

28. Radonovich LJ, Simberkoff MS, Bessesen MT, et al. N95 respirators vs medical masks for preventing influenza among health care personnel: a randomized clinical trial. JAMA 2019;322:824-33.

29. Chu DK, Akl E, Duda S, et al. Physical distancing, face masks, and eye protection to prevent person-to-person transmission of SARS-CoV-2 and COVID-19: a systematic review and meta-analysis. Lancet 2020 June 1 [Epub ahead of print]. doi: 10.1016/S0140-6736(20)31142-9.

30. Mandavilli A. Medical workers should use respirator masks, not surgical masks. New York Times 2020 June $1 .$.

31. Ng K, Poon BH, Kiat Puar TH, et al. COVID-19 and the risk to health care workers: a case report. Ann Intern Med 2020;172:766-7.

32. Heinzerling A, Stuckey MJ, Scheuer T, et al. Transmission of COVID-19 to health care personnel during exposures to a hospitalized patient - Solano County, California, February 2020. MMWR Morb Mortal Wkly Rep 2020;69:472-6. 
33. Tran K, Cimon K, Severn M, et al. Aerosol generating procedures and risk of transmission of acute respiratory infections to healthcare workers: a systematic review. PLoS One 2012; 7:e3579.

34. Tsai YH, Wan GH, Wu YK, et al. Airborne severe acute respiratory syndrome coronavirus concentrations in a negative-pressure isolation room. Infect Control Hosp Epidemiol 2006;27:523-5.

35. Alhazzani W, Moller MH, Arabi YM, et al. Surviving Sepsis Campaign: guidelines on the management of critically ill adults with coronavirus disease 2019 (COVID-19). Intensive Care Med 2020;46:854-87.

36. Mitchell R, Ogunremi T, Astrakianakis G, et al. Impact of the 2009 influenza A (H1N1) pandemic on Canadian health care workers: a survey on vaccination, illness, absenteeism, and personal protective equipment. Am J Infect Control 2012;40:611-6.

37. Patel A, D'Alessandro MM, Ireland KJ, et al. Personal protective equipment supply chain: lessons learned from recent public health emergency responses. Health Secur 2017;15:244-52.

38. Mendoza M, Linderman J. Imports of medical supplies plummet as demand in US soars. Associated Press Mars 2020.

39. Section 2: Evaluation of the National Emergency Stockpile System (NESS) background. Ottawa: Public Health Agency of Canada; 2012. Accessible ici : https://canada.ca/en/public-health/corporate/mandate/about-agency/office -evaluation/evaluation-reports/evaluation-national-emergency-stockpile -system/background-context.html\#background-2 (consulté le 17 mai 2020).

40. Decontamination and reuse of filtering facepiece respirators. Atlanta : Centers for Disease Control and Prevention; 2020. Accessible ici : www.cdc.gov/ coronavirus/2019-ncov/hcp/ppe-strategy/decontamination-reuse-respirators. html (consulté le 10 mai 2020).

41. Decontamination methods for $3 \mathrm{M}$ filtering facepiece respirators such as $\mathrm{N} 95$ respirators. Saint Paul (Minn.) : 3M; 2020. Accessible ici : multimedia.3m.com/ mws/media/18248690/decontamination-methods-for-3m-filtering-facepiece -respirators-technical-bulletin.pdf (consulté le 20 mai 2020).

42. Fischer R, Morris D, van Doremalen N, et al. Assessment of N95 respirator decontamination and re-use for SRAS-CoV-2. medRxiv 2020 Apr. 24. doi: 10.1101/2020.04.11.20062018.

43. DeMaND Study Partners. DeMaND study. Stanford (Cali.): Stanford University; 2020 Accessible ici : stanfordmedicine.app.box.com/s/ s6niip404em07coszrciumjlyneyd6ft (consulté le 1er juin 2020).

44. De Patie M. Manitoba business busy producing 500K reusable N95 masks. CTV News 2020 May 9. Accessible ici : winnipeg.ctvnews.ca/manitoba-business -busy-producing-500k-reusable- n95-masks-1.4932497 (consulté le 17 mai 2020).

45. Casalino E, Astocondor E, Sanchez JC, et al. Personal protective equipment for the Ebola virus disease: a comparison of 2 training programs. Am J Infect Control 2015;43:1281-7.

46. Tong PS, Kale AS, Ng K, et al. Respiratory consequences of N95-type mask usage in pregnant healthcare workers-a controlled clinical study. Antimicrob Resist Infect Control 2015;4:48.

47. Lee HP, Wang De Y. Objective assessment of increase in breathing resistance of N95 respirators on human subjects. Ann Occup Hyg 2011;55:917-21.

48. Roberge RJ, Coca A, Williams WJ, et al. Physiological impact of the N95 filtering facepiece respirator on healthcare workers. Respir Care 2010;55:569-77.

49. Li Y, Tokura H, Guo YP, et al. Effects of wearing N95 and surgical facemasks on heart rate, thermal stress and subjective sensations. Int Arch Occup Environ Health 2005;78:501-9.

50. Foo CC, Goon AT, Leow YH, et al. Adverse skin reactions to personal protective equipment against severe acute respiratory syndrome - a descriptive study in Singapore. Contact Dermatitis 2006;55:291-4

51. Suen LKP, Guo YP, Tong DWK, et al. Self-contamination during doffing of personal protective equipment by healthcare workers to prevent Ebola transmission. Antimicrob Resist Infect Control 2018;7:157.

52. Zamora JE, Murdoch J, Simchison B, et al. Contamination: a comparison of 2 personal protective systems. CMAJ 2006;175:249-54.

53. Fix GM, Reisinger HS, Etchin A, et al. Health care workers' perceptions and reported use of respiratory protective equipment: a qualitative analysis. $\mathrm{Am} J$ Infect Control 2019;47:1162-6.

54. Blackwell T. Canadian nurses treating COVID-19 patients cite unsafe-work laws to demand N95 masks. National Post [Toronto] 2020 Mar. 31.
55. Weeks C. Ontario, Alberta change policy limiting N95 masks as health-care workers demand greater access. The Globe and Mail [Toronto] 2020 Apr. 2.

56. Choi BC. Understanding the basic principles of knowledge translation. $J$ Epidemiol Community Health 2005;59:93.

57. Jull J, Giles A, Graham ID. Community-based participatory research and integrated knowledge translation: advancing the co-creation of knowledge. Implement Sci 2017;12:150.

58. Upshur R, Faith K, Gibson J, et al. Stand on guard for thee: ethical consider ations in preparedness planning for pandemic influenza. Toronto: University of Toronto Joint Centre for Bioethics; 2005

59. Position statement on COVID-19. Ottawa: Canadian Federation of Nurses Unions; 2020. Accessible ici : https://nursesunions.ca/position-statement-on -covid-19/ (consulté le 10 mai 2020).

60. Nurses' ethical considerations in a pandemic or other emergency. Ottawa: Canadian Nurses Association; 2008.

61. Knowledge translation at CIHR. Ottawa: Canadian Institutes for Health Research; 2016. Accessible ici : https://cihr-irsc.gc.ca/e/29418.html\#1 (consulté le 20 mai 2020)

Intérêts concurrents : Henry T. Stelfox a reçu du financement d'une Bourse salariale de clinicien-chercheur intégré des Instituts de recherche en santé du Canada (IRSC). John Conly a reçu des subventions des IRSC pour des recherches sur les mesures de préparation à la COVID-19 dans les milieux de soins actifs et primaires en Alberta (Canada), et il était le chercheur local principal dans le cadre d'une étude sur un vaccin contre Staphylococcus aureus financée par Pfizer, pour laquelle tout le financement a été versé seulement à l'Université de Calgary. Il a également reçu du financement des Centres for Disease Control and Prevention pour assister à une réunion d'un comité de réflexion sur le contrôle des infections. Aucun autre intérêt concurrent déclaré.

Cet article a été révisé par des pairs.

Affiliations : Service des soins intensifs (Ng-Kamstra, Stelfox, Fiest), École de médecine Cumming, Université de Calgary et Alberta Health Services; Département des sciences de la santé communautaire et Institut O'Brien de santé publique (Stelfox, Fiest), département de psychiatrie et Institut Hotchkiss du cerveau (Fiest), Faculté de médecine (Conly), Département de pathologie et de médecine de laboratoire (Conly) et Département de microbiologie, d'immunologie et des maladies infectieuses, et Institut Snyder des maladies chroniques (Conly), École de médecine Cumming, Université de Calgary, Calgary (Alberta); Faculté des sciences de la santé (Parsons Leigh), École d'administration de la santé, et Département de médecine de soins intensifs, Faculté de médecine (Parsons Leigh), Université Dalhousie, Halifax (Nouvelle-Écosse).

Collaborateurs : Josh Ng-Kamstra a mené l'analyse et a rédigé la première version du manuscrit. Henry T. Stelfox, Kirsten Fiest et John Conly ont interprété les données et ont révisé de façon critique le contenu intellectuel important. Jeanna Parsons Leigh a préparé le protocole de l'étude, a interprété les données, a rédigé la première version du manuscrit et en a révisé le contenu de façon critique. Tous les auteurs ont approuvé la version définitive pour publication et ont accepté de se porter garants de tous les aspects du travail.

Financement : Cet article est financé dans le cadre de la Subvention de fonctionnement : possibilité de financement pour une intervention de recherche rapide contre la COVID-2019 des Instituts de recherche en santé du Canada (numéro de subvention : RN420046 439965), qui a été octroyée à J.P.L.

Correspondance : Jeanna Parsons Leigh, j.parsonsleigh@dal.ca 